\title{
Ionic Liquid Design and Process Simulation for Decarbonization of Shale Gas
}

XinyanLiu, ${ }^{\dagger, \dagger}$ Ying Huang, ${ }^{\dagger}$ Yongsheng Zhao, ${ }^{\dagger}$ RafiqulGani, ${ }^{\S}$ XiangpingZhang, ${ }^{*},{ }^{\prime}$ SuojiangZhang ${ }^{*}, \dagger$

$\dagger$ Beijing Key Laboratory of Ionic Liquids Clean Process, State Key Laboratory of Multiphase

Complex Systems, Key Laboratory of Green Process and Engineering, Institute of Process

Engineering, Chinese Academy of Sciences, Beijing 100190, China

\$ Sino-Danish Center for Education and Research, University of Chinese Academy of Sciences, Beijing 100049, China

$\S$ Department of Chemical \& Biochemical Engineering, Technical University of Denmark, DK 2800 Kgs. Lyngby, Denmark

\section{Supplementary material}




\section{Supporting Information}

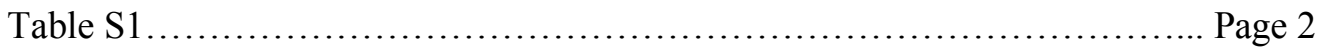

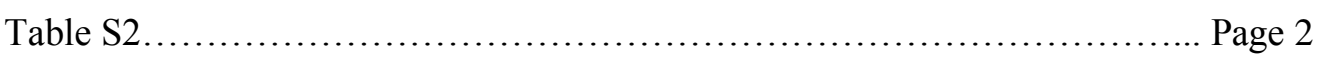

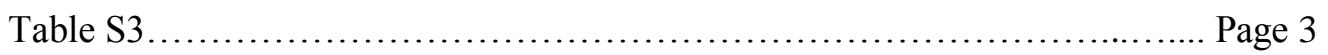

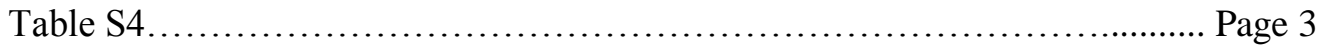

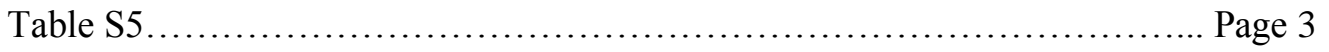

Table S6........................................................ Page 4

Table S7........................................................... Page 4

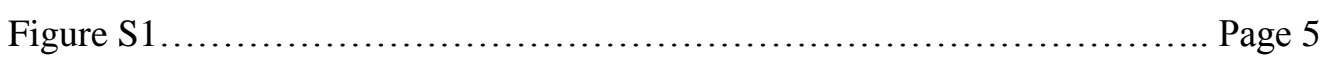

Table S8........................................................... Page $5-10$

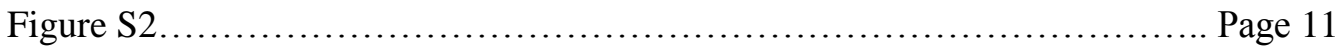

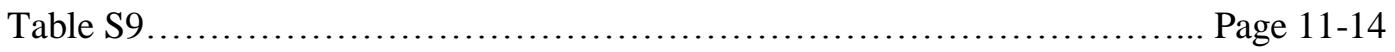

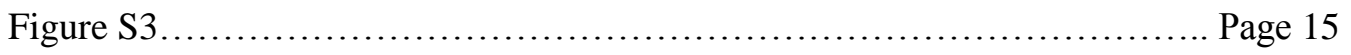

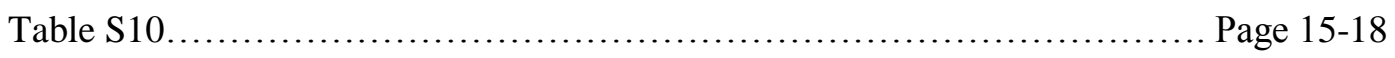

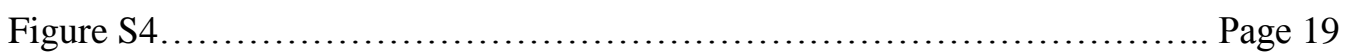

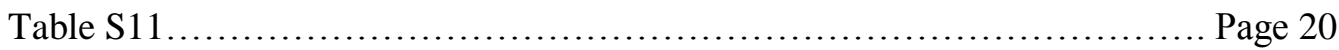

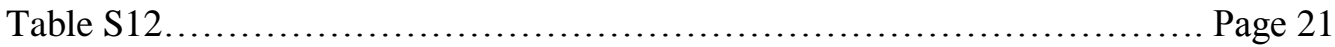

Table S13.......................................................... Page 22

Table S14....................................................... Page 23 
Table $\mathrm{S} 1 \mathrm{CO}_{2}$ henry's constant in 90 kinds of ionic liquids

\begin{tabular}{cccccccccc}
\hline $\mathrm{T}=303.15 \mathrm{~K}$ & {$[\mathrm{HOemim}]$} & {$[\mathrm{Emim}]$} & {$[\mathrm{Bmim}]$} & {$[\mathrm{Hmmim}]$} & {$[\mathrm{Omim}]$} & {$[$ MeButPyrr $]$} & {$[\mathrm{N} 4111]$} & {$[\mathrm{N}-$ bupy $]$} & {$[\mathrm{Hmpy}]$} \\
\hline$[\mathrm{FEP}]$ & 36.37 & 30.83 & 29.03 & 28.11 & 27.56 & 25.91 & 29.80 & 28.13 & 29.52 \\
{$[\mathrm{TCA}]$} & 87.70 & 52.36 & 40.66 & 35.86 & 32.91 & 26.33 & 40.66 & 27.48 & 45.66 \\
{$\left[\mathrm{Tf}_{2} \mathrm{~N}\right]$} & 77.74 & 56.10 & 47.67 & 43.22 & 40.32 & 38.45 & 50.51 & 39.39 & 49.79 \\
{$[\mathrm{DCA}]$} & 102.25 & 57.41 & 49.86 & 44.89 & 41.12 & 30.59 & 49.05 & 33.04 & 56.74 \\
{$[\mathrm{DEP}]$} & 88.45 & 62.95 & 59.90 & 57.29 & 54.71 & 43.71 & 61.48 & 47.48 & 64.85 \\
{$\left[\mathrm{C}_{2} \mathrm{SO}_{4}\right]$} & 110.98 & 70.63 & 65.15 & 60.64 & 56.40 & 45.93 & 67.56 & 49.76 & 70.90 \\
{$[\mathrm{OTF}]$} & 134.95 & 81.09 & 66.01 & 58.06 & 52.69 & 48.54 & 71.91 & 49.33 & 71.29 \\
{$\left[\mathrm{CH}_{3} \mathrm{SO}_{4}\right]$} & 113.59 & 69.02 & 67.88 & 63.75 & 59.17 & 46.27 & 68.38 & 51.42 & 74.41 \\
{$\left[\mathrm{NO}_{3}\right]$} & 103.86 & 64.41 & 71.23 & 68.73 & 63.84 & 45.51 & 66.10 & 53.38 & 79.31 \\
{$\left[\mathrm{BF}_{4}\right]$} & 167.23 & 84.91 & 72.79 & 62.58 & 54.93 & 50.06 & 81.30 & 51.73 & 81.15 \\
\hline
\end{tabular}

Table S2 $\mathrm{CH}_{4}$ henry's constant in 90 kinds of ionic liquids

\begin{tabular}{cccccccccc}
\hline $\mathrm{T}=303.15 \mathrm{~K}$ & {$[\mathrm{HOemim}]$} & {$[\mathrm{Emim}]$} & {$[\mathrm{Bmim}]$} & {$[\mathrm{Hmim}]$} & {$[\mathrm{Omim}]$} & {$[$ MeButPyrr $]$} & {$[\mathrm{N} 4111]$} & {$[\mathrm{N}-b u p y]$} & {$[\mathrm{Hmpy}]$} \\
\hline$[\mathrm{FEP}]$ & 235.90 & 194.52 & 161.24 & 140.45 & 125.88 & 149.40 & 178.08 & 137.03 & 164.52 \\
{$[\mathrm{DEP}]$} & 367.73 & 274.77 & 243.02 & 218.46 & 197.13 & 202.21 & 263.30 & 199.22 & 254.75 \\
{$\left[\mathrm{Tf}_{2} \mathrm{~N}\right]$} & 502.69 & 370.66 & 267.37 & 215.95 & 183.18 & 235.95 & 314.81 & 202.19 & 279.18 \\
{$\left[\mathrm{C}_{2} \mathrm{SO}_{4}\right]$} & 617.49 & 406.42 & 342.79 & 292.89 & 252.00 & 267.19 & 377.36 & 255.97 & 365.60 \\
{$\left[\mathrm{CH}_{3} \mathrm{SO}_{4}\right]$} & 676.38 & 425.57 & 378.50 & 324.12 & 276.08 & 281.87 & 402.68 & 276.53 & 407.39 \\
{$[\mathrm{OTF}]$} & 991.87 & 601.13 & 404.55 & 310.54 & 252.34 & 330.12 & 497.89 & 279.05 & 435.19 \\
{$\left[\mathrm{NO}_{3}\right]$} & 647.75 & 406.42 & 414.45 & 361.12 & 304.45 & 285.99 & 403.05 & 297.65 & 453.01 \\
{$[\mathrm{TCA}]$} & 1155.09 & 706.27 & 434.62 & 321.47 & 255.70 & 331.23 & 516.09 & 272.52 & 483.01 \\
{$[\mathrm{DCA}]$} & 1122.93 & 644.12 & 465.81 & 358.72 & 287.70 & 338.61 & 531.93 & 299.60 & 519.01 \\
{$[\mathrm{BF} 4]$} & 1530.02 & 792.45 & 576.88 & 431.25 & 334.56 & 417.21 & 691.16 & 364.75 & 639.40 \\
\hline
\end{tabular}


Table S3 Scalar property parameters of [bmim] $\left[\mathrm{NTf}_{2}\right]$ IL

\begin{tabular}{ccc}
\hline Parameter & Units & Data \\
\hline Standard boiling point & $\mathrm{K}$ & 739.3 \\
Critical temperature & $\mathrm{K}$ & 1081.4 \\
Critical pressure & $\mathrm{bar}$ & 21.7 \\
Critical volume & $\mathrm{cm}^{3} / \mathrm{mol}$ & 969.9 \\
Acentric factor & $/$ & 0.2316 \\
\hline
\end{tabular}

Table S4 Henry's constant parameters in binary system

\begin{tabular}{ccc}
\hline Parameters & $\mathrm{CO}_{2}$ & $\mathrm{CH}_{4}$ \\
\hline $\mathrm{C}_{1}$ & -322.94 & -267.2 \\
$\mathrm{C}_{2}$ & 3211.152 & 7683.38 \\
$\mathrm{C}_{3}$ & 63.72 & 46.87 \\
$\mathrm{C}_{4}$ & -0.1585 & -0.0658 \\
\hline
\end{tabular}

Table S5 Comparison of predicted value and experimental value for $\mathrm{CO}_{2}$

\begin{tabular}{cccc}
\hline Temperature $(\mathrm{K})$ & Solubility & Predicted P (bar) & Calculated P (bar) \\
\hline 313.15 & 0.0847 & 4.2 & 4.387481 \\
313.15 & 0.1886 & 9.94 & 10.83267 \\
313.15 & 0.2926 & 17.23 & 18.50422 \\
313.15 & 0.3818 & 24.67 & 26.05348 \\
313.15 & 0.4866 & 37.58 & 35.99185 \\
313.15 & 0.5257 & 43.31 & 39.96856 \\
313.15 & 0.5852 & 54.37 & 46.23658 \\
333.15 & 0.0847 & 5.64 & 5.171034 \\
333.15 & 0.1886 & 13.63 & 12.8447 \\
333.15 & 0.2926 & 23.79 & 22.07371 \\
333.15 & 0.3818 & 34.37 & 31.23563 \\
333.15 & 0.4866 & 53.39 & 43.39239 \\
\hline \multicolumn{5}{c}{} \\
\hline
\end{tabular}


Table S6 Comparison of predicted value and experimental value for $\mathrm{CH}_{4}$

\begin{tabular}{cccc}
\hline Temperature $(\mathrm{K})$ & Solubility $\mathrm{x}$ & Predicted P (bar) & Calculated P (bar) \\
313.15 & 0.0298 & 15.01 & 14.10106 \\
313.15 & 0.0555 & 28.51 & 28.03622 \\
313.15 & 0.0911 & 45.76 & 50.00361 \\
313.15 & 0.1222 & 68.56 & 71.64739 \\
313.15 & 0.1628 & 99.12 & 103.1687 \\
333.15 & 0.0298 & 15.91 & 15.61602 \\
333.15 & 0.0555 & 30.63 & 30.79983 \\
333.15 & 0.1222 & 73.2 & 77.43364 \\
333.15 & 0.1628 & 104.97 & 110.6834 \\
\hline
\end{tabular}

Table S7 Energy and solvent consumption of different components of raw gas

\begin{tabular}{ccccc}
\hline $\mathrm{VCO}_{2}: \mathrm{VCH}_{4}$ & $20 \%: 80 \%$ & $40 \%: 60 \%$ & $60 \%: 40 \%$ & $80 \%: 20 \%$ \\
\hline $\mathrm{IL} \mathrm{kg} / \mathrm{h}$ & 850000 & 1100000 & 1484000 & 1898000 \\
$\mathrm{Lean} \mathrm{load}$ & 0.022 & 0.024 & 0.025 & 0.025 \\
$\mathrm{~mol} \mathrm{CO} / \mathrm{mol} \mathrm{IL}$ & 202.67 & 685.85 & 1536.52 & 2442.3 \\
$\mathrm{C} 1 / \mathrm{kW}$ & 580.39 & 738.93 & 979.77 & 1237.9 \\
$\mathrm{P} 1 / \mathrm{kW}$ & 783.06 & 1424.78 & 2516.29 & 3680.2 \\
Electricity (kWe) & 1736.061 & 2257.05 & 3049.61 & 3921.07 \\
Heat duty $(\mathrm{kWth})$ Flash-2 & 1449.71 & 2291.48 & 3687.34 & 5185.89 \\
$\mathrm{TEC} / \mathrm{kW}$ & 0.21 & 0.439 & 1.06 & 2.988 \\
$\mathrm{SEC} / \mathrm{MJ} / \mathrm{kgCH}_{4}$ & 0.34 & 0.245 & 0.256 & 0.267 \\
$\mathrm{SEC} / \mathrm{MJ} / \mathrm{kgCO}_{2}$ & & & & \\
\hline
\end{tabular}




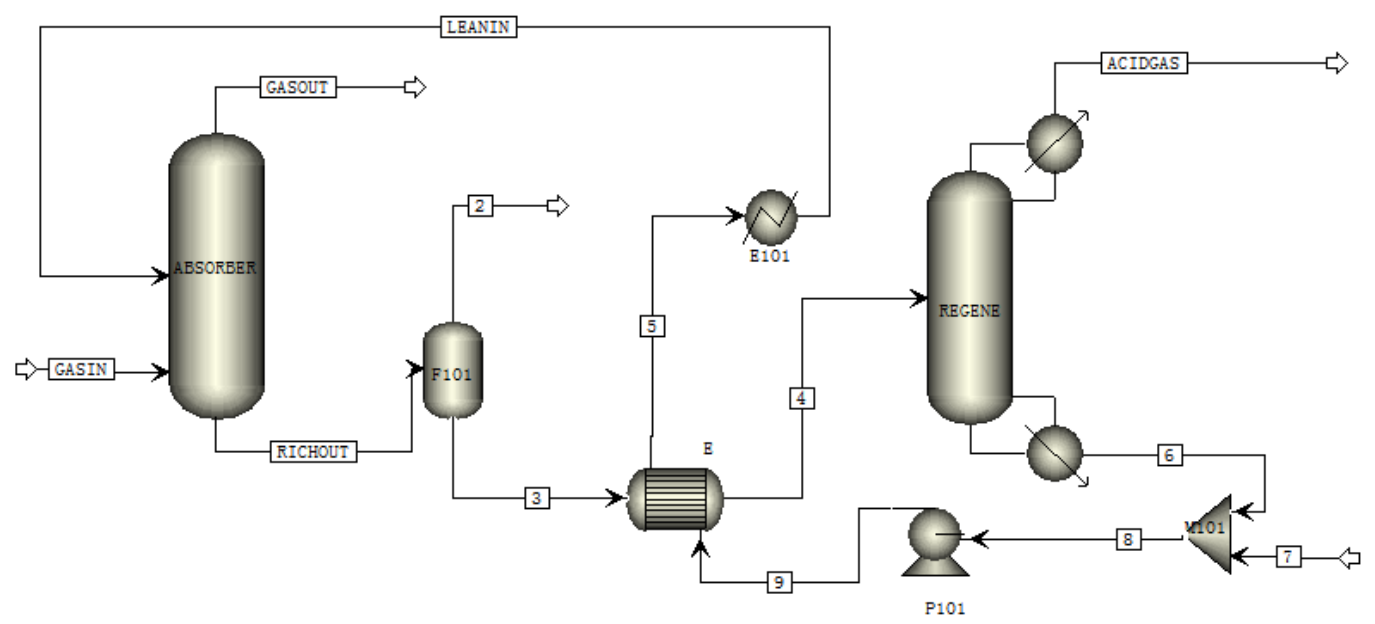

Figure S1 Typical Decarbonization Process Flow Sheet using MDEA Table S8(a) Operation parameters of key equipment in MDEA processes

\begin{tabular}{|c|c|c|}
\hline Items & Unit & Value \\
\hline \multicolumn{3}{|l|}{ Absorber } \\
\hline Pressure of the column bottom & bar & 60 \\
\hline Gas inlet temperature & ${ }^{\circ} \mathrm{C}$ & 20 \\
\hline Gas outlet temperature & ${ }^{\circ} \mathrm{C}$ & 24.5 \\
\hline Stage number & & 7 \\
\hline \multicolumn{3}{|l|}{ Flash (F101) } \\
\hline Pressure & bar & 14 \\
\hline Temperature & ${ }^{\circ} \mathrm{C}$ & 25 \\
\hline \multicolumn{3}{|l|}{ Regenerator } \\
\hline Stage number & & 10 \\
\hline Reflux ratio & & 1.3 \\
\hline Pressure & bar & 1 \\
\hline Top temperature & ${ }^{\circ} \mathrm{C}$ & 102.97 \\
\hline Bottom temperature & ${ }^{\circ} \mathrm{C}$ & 59.5 \\
\hline \multicolumn{3}{|l|}{ Heat exchanger $(\mathrm{E})$} \\
\hline Hot stream inlet temperature & ${ }^{\circ} \mathrm{C}$ & 103.86 \\
\hline Hot stream outlet temperature & ${ }^{\circ} \mathrm{C}$ & 65 \\
\hline Cold stream inlet temperature & ${ }^{\circ} \mathrm{C}$ & 55 \\
\hline Cold stream outlet temperature & ${ }^{\circ} \mathrm{C}$ & 82.7 \\
\hline \multicolumn{3}{|l|}{ Heat exchanger (E101) } \\
\hline Inlet temperature & ${ }^{\circ} \mathrm{C}$ & 65 \\
\hline Outlet temperature & ${ }^{\circ} \mathrm{C}$ & 40 \\
\hline \multicolumn{3}{|l|}{ Purified shale gas } \\
\hline $\mathrm{CH}_{4}$ & mole fraction & 0.846 \\
\hline $\mathrm{CO}_{2}$ & mole fraction & 0.013 \\
\hline
\end{tabular}


Table S8(b) Temperature, Pressure and Vapor and Liquid Flow Rate Profiles of the Absorber of the Modified MDEA Process

\begin{tabular}{ccccc}
\hline Stage & $\begin{array}{c}\text { Temperature } \\
\mathrm{C}\end{array}$ & $\begin{array}{c}\text { Pressure } \\
\text { bar }\end{array}$ & $\begin{array}{c}\text { Liquid from (Mole) } \\
\mathrm{kmol} / \mathrm{hr}\end{array}$ & $\begin{array}{c}\text { Vapor from (Mole) } \\
\mathrm{kmol} / \mathrm{hr}\end{array}$ \\
\hline 1 & 61.54 & 59.98 & 1602.937 & 1892.395 \\
2 & 68.58 & 59.99 & 1605.250 & 1953.547 \\
3 & 69.92 & 60.01 & 1605.806 & 1975.765 \\
4 & 69.85 & 60.02 & 1605.518 & 1980.721 \\
5 & 68.98 & 60.03 & 1604.397 & 1982.093 \\
6 & 66.51 & 60.04 & 1601.674 & 1983.534 \\
7 & 59.58 & 60.05 & 1594.286 & 1987.578 \\
\hline
\end{tabular}

Table S8(c) Vapor and Liquid Composition Profiles of the Absorber of the MDEA Process Vapor (mole fraction)

\begin{tabular}{cccccccccc}
\hline Stage & MDEA & $\mathrm{H}_{2} \mathrm{O}$ & $\mathrm{CO}_{2}$ & $\mathrm{CH}_{4}$ & $\mathrm{~N}_{2}$ & $\mathrm{C}_{2} \mathrm{H}_{6}$ & $\mathrm{C}_{3} \mathrm{H}_{8}$ & $\mathrm{C}_{4} \mathrm{H}_{10-01}$ & $\mathrm{C}_{5} \mathrm{H}_{12-01}$ \\
\hline 1 & $1.50 \mathrm{E}-06$ & 0.005 & 0.013 & 0.846 & 0.029 & 0.069 & 0.020 & 0.014 & 0.003 \\
2 & $2.43 \mathrm{E}-06$ & 0.006 & 0.042 & 0.820 & 0.028 & 0.067 & 0.020 & 0.013 & 0.003 \\
3 & $2.64 \mathrm{E}-06$ & 0.006 & 0.052 & 0.811 & 0.028 & 0.067 & 0.019 & 0.013 & 0.003 \\
4 & $2.61 \mathrm{E}-06$ & 0.006 & 0.055 & 0.809 & 0.028 & 0.066 & 0.019 & 0.013 & 0.003 \\
5 & $2.44 \mathrm{E}-06$ & 0.006 & 0.056 & 0.808 & 0.028 & 0.066 & 0.019 & 0.013 & 0.003 \\
6 & $1.99 \mathrm{E}-06$ & 0.006 & 0.057 & 0.807 & 0.028 & 0.066 & 0.019 & 0.013 & 0.003 \\
7 & $1.08 \mathrm{E}-06$ & 0.004 & 0.060 & 0.806 & 0.028 & 0.066 & 0.019 & 0.013 & 0.003 \\
\hline
\end{tabular}


Liquid (mole fraction)

\begin{tabular}{ccccccccccccccccccc}
\hline Stage & MDEA & $\mathrm{H}_{2} \mathrm{O}$ & $\mathrm{CO}_{2}$ & $\mathrm{H}_{3} \mathrm{O}^{+}$ & $\mathrm{OH}$ & $\mathrm{HCO}_{3}^{-}$ & $\mathrm{CO}_{3}^{-2}$ & $\mathrm{MDEAH}^{+}$ & $\mathrm{CH}_{4}$ & $\mathrm{~N}_{2}$ & $\mathrm{C}_{2} \mathrm{H}_{6}$ & $\mathrm{C}_{3} \mathrm{H}_{8}$ & $\mathrm{C}_{4} \mathrm{H}_{10-01}$ & $\mathrm{C}_{5} \mathrm{H}_{12-01}$ \\
\hline 1 & 0.095971 & 0.8328 & 0.000539 & $1.19 \mathrm{E}-10$ & $2.01 \mathrm{E}-06$ & 0.034865 & $1.51 \mathrm{E}-06$ & 0.03491 & 0.000782 & $1.64 \mathrm{E}-05$ & $5.66 \mathrm{E}-05$ & $1.05 \mathrm{E}-05$ & $4.17 \mathrm{E}-06$ & $8.12 \mathrm{E}-07$ \\
2 & 0.083416 & 0.81967 & 0.001566 & $2.62 \mathrm{E}-10$ & $1.53 \mathrm{E}-06$ & 0.047215 & $5.12 \mathrm{E}-07$ & 0.047277 & 0.000711 & $1.53 \mathrm{E}-05$ & $5.12 \mathrm{E}-05$ & $9.53 \mathrm{E}-06$ & $3.70 \mathrm{E}-06$ & $7.27 \mathrm{E}-07$ \\
3 & 0.080625 & 0.81663 & 0.001924 & $3.02 \mathrm{E}-10$ & $1.44 \mathrm{E}-06$ & 0.049939 & $3.97 \mathrm{E}-07$ & 0.050023 & 0.000694 & $1.50 \mathrm{E}-05$ & $4.99 \mathrm{E}-05$ & $9.30 \mathrm{E}-06$ & $3.60 \mathrm{E}-06$ & $7.06 \mathrm{E}-07$ \\
4 & 0.079573 & 0.815449 & 0.00201 & $3.07 \mathrm{E}-10$ & $1.40 \mathrm{E}-06$ & 0.050982 & $3.67 \mathrm{E}-07$ & 0.051097 & 0.000689 & $1.49 \mathrm{E}-05$ & $4.95 \mathrm{E}-05$ & $9.23 \mathrm{E}-06$ & $3.57 \mathrm{E}-06$ & $7.01 \mathrm{E}-07$ \\
5 & 0.077983 & 0.813634 & 0.002055 & $3.00 \mathrm{E}-10$ & $1.34 \mathrm{E}-06$ & 0.052615 & $3.34 \mathrm{E}-07$ & 0.052778 & 0.000685 & $1.48 \mathrm{E}-05$ & $4.92 \mathrm{E}-05$ & $9.16 \mathrm{E}-06$ & $3.55 \mathrm{E}-06$ & $6.97 \mathrm{E}-07$ \\
6 & 0.073811 & 0.808937 & 0.002143 & $2.76 \mathrm{E}-10$ & $1.20 \mathrm{E}-06$ & 0.056929 & $2.63 \mathrm{E}-07$ & 0.057172 & 0.000677 & $1.44 \mathrm{E}-05$ & $4.87 \mathrm{E}-05$ & $9.02 \mathrm{E}-06$ & $3.53 \mathrm{E}-06$ & $6.90 \mathrm{E}-07$ \\
7 & 0.06157 & 0.795247 & 0.002388 & $2.17 \mathrm{E}-10$ & $8.65 \mathrm{E}-07$ & 0.069619 & $1.26 \mathrm{E}-07$ & 0.070018 & 0.000659 & $1.37 \mathrm{E}-05$ & $4.76 \mathrm{E}-05$ & $8.75 \mathrm{E}-06$ & $3.49 \mathrm{E}-06$ & $6.76 \mathrm{E}-07$ \\
\hline
\end{tabular}


Table S8(d) Temperature, Pressure and Vapor and Liquid Flow Rate Profiles of the Regenerator of the Modified MDEA Process

\begin{tabular}{cccccc}
\hline Stage & $\begin{array}{c}\text { Temperature } \\
\text { Pressure }\end{array}$ & $\begin{array}{c}\text { Heat duty } \\
\text { bar }\end{array}$ & $\begin{array}{c}\text { Liquid from } \\
\text { Wott }\end{array}$ & $\begin{array}{c}\text { Vapor from } \\
\text { kmol/hr }\end{array}$ & $\begin{array}{c}\text { (Mole) } \\
\mathrm{kmol} / \mathrm{hr}\end{array}$ \\
\hline 1 & 70.19 & 0.98 & -2627233 & 218.3313 & 167.9471 \\
2 & 89.99 & 0.99 & 0 & 226.1483 & 386.2786 \\
3 & 90.21 & 1.01 & 0 & 226.2335 & 394.0956 \\
4 & 90.23 & 1.02 & 0 & 225.8344 & 394.1808 \\
5 & 97.71 & 1.03 & 0 & 1963.239 & 305.6293 \\
6 & 101.79 & 1.04 & 0 & 1991.162 & 435.3079 \\
7 & 102.16 & 1.05 & 0 & 1994.495 & 457.4142 \\
8 & 102.22 & 1.06 & 0 & 1995.263 & 459.6779 \\
9 & 102.23 & 1.07 & 0 & 1995.224 & 460.1286 \\
10 & 103.02 & 1.08 & 5324023.8 & 1535.442 & 459.9438 \\
\hline
\end{tabular}

Table S8(e) Vapor and Liquid Composition Profiles of the Regenerator of the MDEA Process

Vapor (mole fraction)

\begin{tabular}{cccccccccc}
\hline Stage & MDEA & $\mathrm{H}_{2} \mathrm{O}$ & $\mathrm{CO}_{2}$ & $\mathrm{CH}_{4}$ & $\mathrm{~N}_{2}$ & $\mathrm{C}_{2} \mathrm{H}_{6}$ & $\mathrm{C}_{3} \mathrm{H}_{8}$ & $\mathrm{C}_{4} \mathrm{H}_{10-01}$ & $\mathrm{C}_{5} \mathrm{H}_{12-01}$ \\
\hline 1 & $1.29 \mathrm{E}-25$ & 0.317 & 0.679 & 0.000492 & $5.74 \mathrm{E}-06$ & $3.88 \mathrm{E}-05$ & $5.42 \mathrm{E}-06$ & $1.54 \mathrm{E}-06$ & $2.94 \mathrm{E}-07$ \\
2 & $1.34 \mathrm{E}-18$ & 0.703 & 0.295 & 0.000214 & $2.50 \mathrm{E}-06$ & $1.69 \mathrm{E}-05$ & $2.36 \mathrm{E}-06$ & $6.70 \mathrm{E}-07$ & $1.28 \mathrm{E}-07$ \\
3 & $5.70 \mathrm{E}-13$ & 0.709 & 0.289 & 0.00021 & $2.45 \mathrm{E}-06$ & $1.65 \mathrm{E}-05$ & $2.31 \mathrm{E}-06$ & $6.57 \mathrm{E}-07$ & $1.25 \mathrm{E}-07$ \\
4 & $1.82 \mathrm{E}-07$ & 0.709 & 0.289 & 0.00021 & $2.45 \mathrm{E}-06$ & $1.65 \mathrm{E}-05$ & $2.31 \mathrm{E}-06$ & $6.57 \mathrm{E}-07$ & $1.25 \mathrm{E}-07$ \\
5 & 0.000291 & 0.842 & 0.156 & $8.83 \mathrm{E}-08$ & $5.85 \mathrm{E}-10$ & $7.51 \mathrm{E}-09$ & $7.90 \mathrm{E}-10$ & $1.50 \mathrm{E}-10$ & $2.84 \mathrm{E}-11$ \\
6 & 0.000376 & 0.982 & 0.018 & $7.66 \mathrm{E}-12$ & $3.15 \mathrm{E}-14$ & $6.82 \mathrm{E}-13$ & $5.28 \mathrm{E}-14$ & $5.89 \mathrm{E}-15$ & $1.11 \mathrm{E}-15$ \\
7 & 0.000384 & 0.996 & 0.004 & $6.52 \mathrm{E}-16$ & $1.68 \mathrm{E}-18$ & $6.05 \mathrm{E}-17$ & $3.43 \mathrm{E}-18$ & $2.21 \mathrm{E}-19$ & $4.17 \mathrm{E}-20$ \\
8 & 0.000385 & 0.998 & 0.001 & $5.54 \mathrm{E}-20$ & $1.06 \mathrm{E}-22$ & $5.29 \mathrm{E}-21$ & $2.34 \mathrm{E}-22$ & $8.29 \mathrm{E}-24$ & $1.16 \mathrm{E}-25$ \\
9 & 0.000386 & 0.999 & 0.001 & $4.70 \mathrm{E}-24$ & $3.99 \mathrm{E}-23$ & $4.90 \mathrm{E}-26$ & $6.70 \mathrm{E}-26$ & $1.16 \mathrm{E}-25$ & $1.16 \mathrm{E}-25$ \\
10 & 0.000496 & 0.999 & 0.000 & $4.88 \mathrm{E}-26$ & $7.76 \mathrm{E}-26$ & $1.74 \mathrm{E}-23$ & $1.95 \mathrm{E}-23$ & $1.11 \mathrm{E}-25$ & $1.83 \mathrm{E}-19$ \\
\hline
\end{tabular}


Liquid (mole fraction)

\begin{tabular}{ccccccccccccccccc}
\hline Stage & MDEA & $\mathrm{H}_{2} \mathrm{O}$ & $\mathrm{CO}_{2}$ & $\mathrm{H}_{3} \mathrm{O}^{+}$ & $\mathrm{OH}^{-}$ & $\mathrm{HCO}_{3}^{-}$ & $\mathrm{CO}_{3}^{-2}$ & MDEAH $^{+}$ & $\mathrm{CH}_{4}$ & $\mathrm{~N}_{2}$ & $\mathrm{C}_{2} \mathrm{H}_{6}$ & $\mathrm{C}_{3} \mathrm{H}_{8}$ & $\mathrm{C}_{4} \mathrm{H}_{10-01}$ & $\mathrm{C}_{5} \mathrm{H}_{12-01}$ \\
\hline 1 & $3.17 \mathrm{E}-22$ & 1.000 & 0.000169 & $1.16 \mathrm{E}-06$ & $4.13 \mathrm{E}-11$ & $1.15 \mathrm{E}-06$ & $1.23 \mathrm{E}-12$ & $2.36 \mathrm{E}-18$ & $7.42 \mathrm{E}-09$ & $4.94 \mathrm{E}-11$ & $6.29 \mathrm{E}-10$ & $6.62 \mathrm{E}-11$ & $1.25 \mathrm{E}-11$ & $2.37 \mathrm{E}-12$ \\
2 & $6.13 \mathrm{E}-16$ & 1.000 & $5.90 \mathrm{E}-05$ & $6.32 \mathrm{E}-07$ & $1.74 \mathrm{E}-10$ & $6.21 \mathrm{E}-07$ & $1.09 \mathrm{E}-12$ & $9.93 \mathrm{E}-13$ & $3.05 \mathrm{E}-09$ & $2.16 \mathrm{E}-11$ & $2.53 \mathrm{E}-10$ & $2.63 \mathrm{E}-11$ & $4.55 \mathrm{E}-12$ & $8.63 \mathrm{E}-13$ \\
3 & $2.57 \mathrm{E}-10$ & 1.000 & $5.77 \mathrm{E}-05$ & $4.86 \mathrm{E}-07$ & $2.28 \mathrm{E}-10$ & $7.89 \mathrm{E}-07$ & $1.81 \mathrm{E}-12$ & $3.17 \mathrm{E}-07$ & $2.99 \mathrm{E}-09$ & $2.12 \mathrm{E}-11$ & $2.48 \mathrm{E}-10$ & $2.58 \mathrm{E}-11$ & $4.45 \mathrm{E}-12$ & $8.44 \mathrm{E}-13$ \\
4 & $8.19 \mathrm{E}-05$ & 0.999 & $5.79 \mathrm{E}-05$ & $1.59 \mathrm{E}-09$ & $9.39 \mathrm{E}-08$ & 0.000322 & $3.83 \mathrm{E}-07$ & 0.000328 & $2.98 \mathrm{E}-09$ & $2.12 \mathrm{E}-11$ & $2.48 \mathrm{E}-10$ & $2.57 \mathrm{E}-11$ & $4.45 \mathrm{E}-12$ & $8.43 \mathrm{E}-13$ \\
5 & 0.102947 & 0.889 & $8.27 \mathrm{E}-05$ & $1.36 \mathrm{E}-10$ & $8.43 \mathrm{E}-06$ & 0.003913 & $2.90 \mathrm{E}-06$ & 0.003995 & $1.70 \mathrm{E}-12$ & $6.98 \mathrm{E}-15$ & $1.51 \mathrm{E}-13$ & $1.17 \mathrm{E}-14$ & $1.31 \mathrm{E}-15$ & $2.47 \mathrm{E}-16$ \\
6 & 0.104449 & 0.894 & $9.00 \mathrm{E}-06$ & $4.12 \mathrm{E}-11$ & $2.26 \mathrm{E}-05$ & 0.000939 & $1.44 \mathrm{E}-06$ & 0.000999 & $1.50 \mathrm{E}-16$ & $3.85 \mathrm{E}-19$ & $1.39 \mathrm{E}-17$ & $7.87 \mathrm{E}-19$ & $5.08 \mathrm{E}-20$ & $9.58 \mathrm{E}-21$ \\
7 & 0.104797 & 0.894 & $1.90 \mathrm{E}-06$ & $1.73 \mathrm{E}-11$ & $4.64 \mathrm{E}-05$ & 0.000402 & $1.10 \mathrm{E}-06$ & 0.000476 & $1.28 \mathrm{E}-20$ & $2.06 \mathrm{E}-23$ & $1.23 \mathrm{E}-21$ & $5.12 \mathrm{E}-23$ & $1.91 \mathrm{E}-24$ & $3.58 \mathrm{E}-25$ \\
8 & 0.104889 & 0.894 & $6.95 \mathrm{E}-07$ & $9.87 \mathrm{E}-12$ & $7.66 \mathrm{E}-05$ & 0.000242 & $1.03 \mathrm{E}-06$ & 0.000343 & $1.08 \mathrm{E}-24$ & $1.30 \mathrm{E}-27$ & $1.08 \mathrm{E}-25$ & $3.49 \mathrm{E}-27$ & $7.15 \mathrm{E}-29$ & $1.00 \mathrm{E}-30$ \\
9 & 0.104959 & 0.894 & $3.40 \mathrm{E}-07$ & $6.77 \mathrm{E}-12$ & 0.000109 & 0.000169 & $1.00 \mathrm{E}-06$ & 0.000302 & $9.20 \mathrm{E}-29$ & $4.90 \mathrm{E}-28$ & $1.00 \mathrm{E}-30$ & $1.00 \mathrm{E}-30$ & $1.00 \mathrm{E}-30$ & $1.00 \mathrm{E}-30$ \\
10 & 0.13632 & 0.863 & $2.24 \mathrm{E}-07$ & $4.69 \mathrm{E}-12$ & 0.000172 & 0.000116 & $2.48 \mathrm{E}-07$ & 0.000311 & $1.00 \mathrm{E}-30$ & $1.00 \mathrm{E}-30$ & $3.72 \mathrm{E}-28$ & $3.05 \mathrm{E}-28$ & $1.00 \mathrm{E}-30$ & $1.64 \mathrm{E}-24$ \\
\hline
\end{tabular}


Table S8(f) Stream results of the flowsheet for MDEA Decarbonization

\begin{tabular}{|c|c|c|c|c|c|c|c|c|c|c|c|c|c|}
\hline & 2 & 3 & 4 & 5 & 6 & 7 & 8 & 9 & ACIDGAS & GASIN & GASOUT & LEANIN & RICHOUT \\
\hline Temperature ${ }^{\circ} \mathrm{C}$ & 55.00 & 55.00 & 82.67 & 65.00 & 103.02 & 20.00 & 101.06 & 103.14 & 70.19 & 40.00 & 61.54 & 40.00 & 59.58 \\
\hline Pressure bar & 5.70 & 5.70 & 5.70 & 60.00 & 1.00 & 1.00 & 1.00 & 60.00 & 1.00 & 60.00 & 60.00 & 60.00 & 60.00 \\
\hline Vapor Frac & 1.00 & 0.00 & 0.02 & 0.00 & 0.00 & 0.00 & 0.00 & 0.00 & 1.00 & 1.00 & 1.00 & 0.00 & 0.00 \\
\hline Mass Flow kg/hr & 46.04 & 54889.6 & 54889.6 & 50006.6 & 48889.65 & 1117.0 & 50006.6 & 50006.6 & 6000.00 & 41038.3 & 36110.49 & 50007.8 & 54935.69 \\
\hline Volume Flow cum $/ \mathrm{hr}$ & 8.08 & 50.25 & 233.06 & 49.55 & 50.04 & 1.12 & 51.11 & 51.13 & 4771.40 & 760.19 & 797.75 & 48.70 & 50.55 \\
\hline Enthalpy Gcal/hr & -0.08 & -129.39 & -127.81 & -117.47 & -111.76 & -4.24 & -116.00 & -115.89 & -13.73 & -46.56 & -35.78 & -118.48 & -129.26 \\
\hline Density kg/cum & 5.70 & 1092.37 & 235.52 & 1009.24 & 977.09 & 998.33 & 978.48 & 978.11 & 1.26 & 53.98 & 45.27 & 1026.87 & 1086.71 \\
\hline Mass Flow MDEA kg/hr & 0.00 & 11658.4 & 15923.9 & 24948.1 & 24942.38 & 0.00 & 24940.9 & 24940.7 & 0.00 & 0.00 & 0.34 & 24955.7 & 11697.19 \\
\hline $\mathrm{H}_{2} \mathrm{O} \mathrm{kg} / \mathrm{hr}$ & 0.68 & 22834.1 & 23477.4 & 24991.3 & 23873.48 & 1117.0 & 24990.2 & 24990.2 & 958.14 & 1.62 & 160.66 & 24993.2 & 22840.69 \\
\hline $\mathrm{CO}_{2} \mathrm{~kg} / \mathrm{hr}$ & 26.13 & 127.19 & 1698.61 & 0.00 & 0.02 & 0.00 & 0.01 & 0.01 & 5018.30 & 6160.79 & 1116.36 & 0.00 & 167.54 \\
\hline $\mathrm{H}_{3} \mathrm{O}^{+} \mathrm{kg} / \mathrm{hr}$ & 0.00 & 0.00 & 0.00 & 0.00 & 0.00 & 0.00 & 0.00 & 0.00 & 0.00 & 0.00 & 0.00 & 0.00 & 0.00 \\
\hline OH- kg/hr & 0.00 & 0.02 & 0.04 & 3.72 & 4.50 & 0.00 & 4.70 & 4.74 & 0.00 & 0.00 & 0.00 & 2.68 & 0.02 \\
\hline $\mathrm{HCO}^{3-} \mathrm{kg} / \mathrm{hr}$ & 0.00 & 6792.20 & 4613.47 & 10.80 & 10.85 & 0.00 & 10.84 & 10.84 & 0.00 & 0.00 & 0.00 & 10.72 & 6772.48 \\
\hline $\mathrm{CO}_{3}^{-2} \mathrm{~kg} / \mathrm{hr}$ & 0.00 & 0.01 & 0.03 & 0.09 & 0.02 & 0.00 & 0.03 & 0.03 & 0.00 & 0.00 & 0.00 & 0.17 & 0.01 \\
\hline $\mathrm{MDEAH}^{+} \mathrm{kg} / \mathrm{hr}$ & 0.00 & 13453.6 & 9152.15 & 51.59 & 57.43 & 0.00 & 58.82 & 59.11 & 0.00 & 0.00 & 0.00 & 44.29 & 13414.59 \\
\hline $\mathrm{CH}_{4} \mathrm{~kg} / \mathrm{hr}$ & 15.53 & 1.33 & 1.33 & 0.00 & 0.00 & 0.00 & 0.00 & 0.00 & 1.33 & 25693.9 & 25677.09 & 0.00 & 16.85 \\
\hline $\mathrm{N}_{2} \mathrm{~kg} / \mathrm{hr}$ & 0.59 & 0.03 & 0.03 & 0.00 & 0.00 & 0.00 & 0.00 & 0.00 & 0.03 & 1555.84 & 1555.22 & 0.00 & 0.61 \\
\hline $\mathrm{C}_{2} \mathrm{H}_{6} \mathrm{~kg} / \mathrm{hr}$ & 2.09 & 0.20 & 0.20 & 0.00 & 0.00 & 0.00 & 0.00 & 0.00 & 0.20 & 3955.66 & 3953.38 & 0.00 & 2.28 \\
\hline $\mathrm{C}_{3} \mathrm{H}_{8} \mathrm{~kg} / \mathrm{hr}$ & 0.57 & 0.04 & 0.04 & 0.00 & 0.00 & 0.00 & 0.00 & 0.00 & 0.04 & 1692.66 & 1692.05 & 0.00 & 0.61 \\
\hline $\mathrm{C}_{4} \mathrm{H}_{10-01} \mathrm{~kg} / \mathrm{hr}$ & 0.31 & 0.02 & 0.02 & 0.00 & 0.00 & 0.00 & 0.00 & 0.00 & 0.02 & 1522.25 & 1521.93 & 0.00 & 0.32 \\
\hline $\mathrm{C}_{5} \mathrm{H}_{12-01} \mathrm{~kg} / \mathrm{hr}$ & 0.07 & 0.00 & 0.00 & 0.00 & 0.00 & 0.00 & 0.00 & 0.00 & 0.00 & 432.74 & 432.66 & 0.00 & 0.08 \\
\hline
\end{tabular}




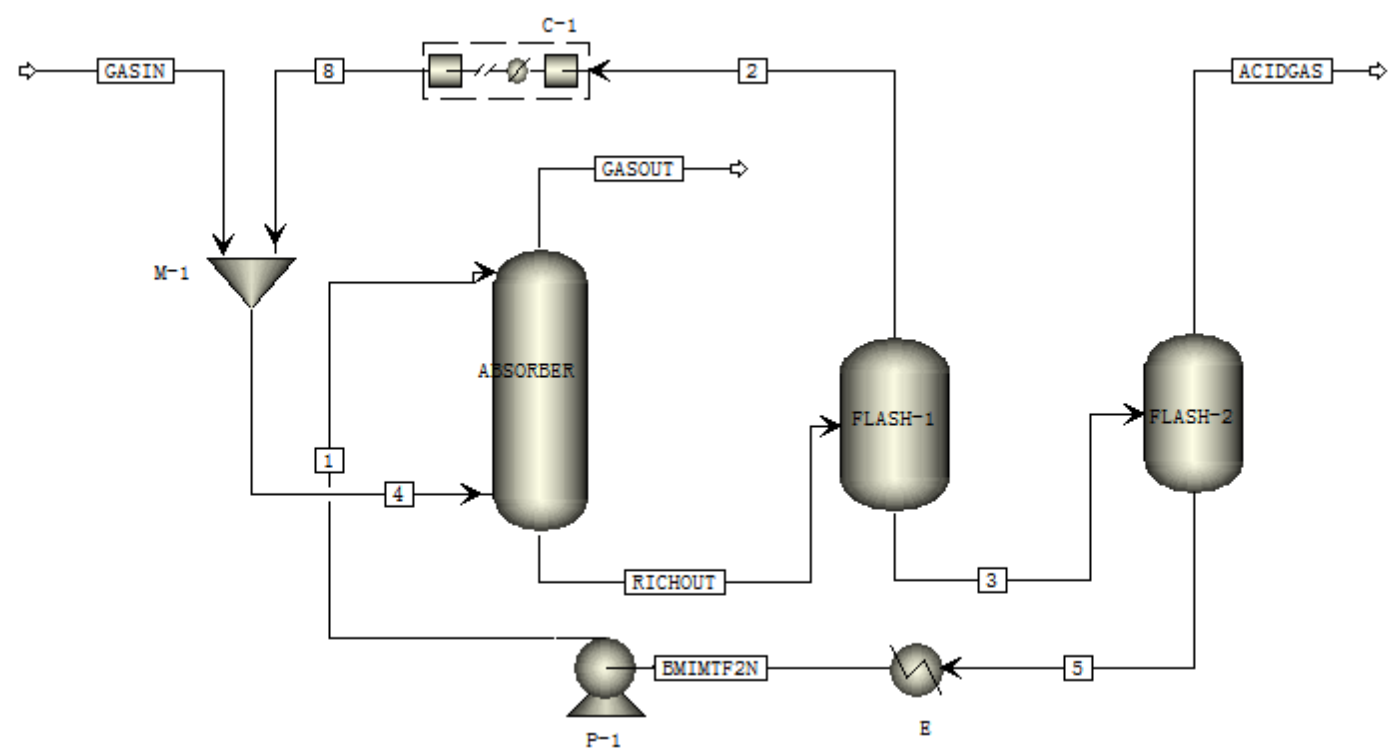

Figure S2 IL-based Decarbonization Flow Sheet (two single-stage)

Table S9(a) Operation parameters of key equipment in IL-based decarbonization process (two single-stage)

\begin{tabular}{lll}
\hline Items & Unit & Value \\
\hline Absorber & & \\
Pressure of the column bottom & bar & 60 \\
Gas inlet temperature & ${ }^{\circ} \mathrm{C}$ & 20 \\
Gas outlet temperature & ${ }^{\circ} \mathrm{C}$ & 24.5 \\
Stage number & & 10 \\
Flash-1 & & \\
Pressure & bar & 15 \\
Temperature & ${ }^{\circ} \mathrm{C}$ & 25 \\
Flash-2 & & \\
Pressure & bar & 1 \\
Temperature & ${ }^{\circ} \mathrm{C}$ & 27 \\
Heat exchanger (E) & & \\
Inlet temperature & ${ }^{\circ} \mathrm{C}$ & 27 \\
Outlet temperature & ${ }^{\circ} \mathrm{C}$ & 20 \\
Purified shale gas & & \\
$\mathrm{CH}_{4}$ & mole fraction & 0.848 \\
$\mathrm{CO}_{2}$ & mole fraction & 0.012 \\
\hline
\end{tabular}


Table S9(b) Temperature, Pressure and Vapor and Liquid Flow Rate Profiles of the Absorber of the IL-based Decarbonization Process (two single-stage)

\begin{tabular}{ccccc}
\hline Stage & $\begin{array}{c}\text { Temperature } \\
\mathrm{C}\end{array}$ & $\begin{array}{c}\text { Pressure } \\
\text { bar }\end{array}$ & $\begin{array}{c}\text { Liquid from (Mole) } \\
\mathrm{kmol} / \mathrm{hr}\end{array}$ & $\begin{array}{c}\text { Vapor from (Mole) } \\
\mathrm{kmol} / \mathrm{hr}\end{array}$ \\
\hline 1 & 23.16 & 59.98 & 1620.269 & 1838.362 \\
2 & 23.26 & 59.99 & 1627.622 & 1998.135 \\
3 & 23.38 & 60.01 & 1635.167 & 2005.488 \\
4 & 23.55 & 60.02 & 1645.048 & 2013.033 \\
5 & 23.76 & 60.03 & 1657.809 & 2022.914 \\
6 & 24.02 & 60.04 & 1673.95 & 2035.675 \\
7 & 24.35 & 60.05 & 1693.855 & 2051.816 \\
8 & 24.73 & 60.06 & 1717.683 & 2071.721 \\
9 & 25.15 & 60.07 & 1745.296 & 2095.549 \\
10 & 25.47 & 60.08 & 1777.967 & 2123.162 \\
\hline
\end{tabular}

Table S9(c) Vapor and Liquid Composition Profiles of the Absorber of the IL-based Decarbonization Process (two single-stage)

Vapor (mole fraction)

\begin{tabular}{cccccccccc}
\hline Stage & $\mathrm{CO}_{2}$ & $\mathrm{BMIMTF}_{2} \mathrm{~N}$ & $\mathrm{CH}_{4}$ & $\mathrm{C}_{2} \mathrm{H}_{6}$ & $\mathrm{C}_{3} \mathrm{H}_{8}$ & $\mathrm{~N}_{2}$ & $\mathrm{H}_{2} \mathrm{O}$ & $\mathrm{C}_{4} \mathrm{H}_{10}$ & $\mathrm{C}_{5} \mathrm{H}_{12}$ \\
\hline 1 & 0.011927 & $6.37 \mathrm{E}-25$ & 0.847905 & 0.071561 & 0.020881 & 0.030212 & $5.33 \mathrm{E}-08$ & 0.014247 & 0.003263 \\
2 & 0.013917 & $6.16 \mathrm{E}-25$ & 0.856949 & 0.065952 & 0.019238 & 0.027813 & $5.37 \mathrm{E}-08$ & 0.013122 & 0.003005 \\
3 & 0.016586 & $5.88 \mathrm{E}-25$ & 0.854767 & 0.065701 & 0.019165 & 0.02771 & $5.43 \mathrm{E}-08$ & 0.013073 & 0.002994 \\
4 & 0.020114 & $5.54 \mathrm{E}-25$ & 0.851722 & 0.065454 & 0.019094 & 0.027606 & $5.50 \mathrm{E}-08$ & 0.013024 & 0.002983 \\
5 & 0.024707 & $5.15 \mathrm{E}-25$ & 0.847756 & 0.065134 & 0.019 & 0.027471 & $5.60 \mathrm{E}-08$ & 0.01296 & 0.002968 \\
6 & 0.030573 & $4.73 \mathrm{E}-25$ & 0.842691 & 0.064724 & 0.018881 & 0.027299 & $5.72 \mathrm{E}-08$ & 0.012879 & 0.002949 \\
7 & 0.03789 & $4.28 \mathrm{E}-25$ & 0.836371 & 0.064214 & 0.018732 & 0.027084 & $5.88 \mathrm{E}-08$ & 0.012777 & 0.002926 \\
8 & 0.046763 & $3.83 \mathrm{E}-25$ & 0.828707 & 0.063596 & 0.018552 & 0.026823 & $6.07 \mathrm{E}-08$ & 0.012654 & 0.002898 \\
9 & 0.057172 & $3.42 \mathrm{E}-25$ & 0.819714 & 0.062872 & 0.01834 & 0.026518 & $6.30 \mathrm{E}-08$ & 0.01251 & 0.002865 \\
10 & 0.06895 & $3.03 \mathrm{E}-25$ & 0.809533 & 0.062052 & 0.018101 & 0.026173 & $6.49 \mathrm{E}-08$ & 0.012347 & 0.002828 \\
\hline
\end{tabular}


Liquid (mole fraction)

\begin{tabular}{ccccccccccc}
\hline Stage & $\mathrm{CO}_{2}$ & $\mathrm{BMIMTF}_{2} \mathrm{~N}$ & $\mathrm{CH}_{4}$ & $\mathrm{C}_{2} \mathrm{H}_{6}$ & $\mathrm{C}_{3} \mathrm{H}_{8}$ & $\mathrm{~N}_{2}$ & $\mathrm{H}_{2} \mathrm{O}$ & $\mathrm{C}_{4} \mathrm{H}_{10}$ & $\mathrm{C}_{5} \mathrm{H}_{12}$ \\
\hline 1 & 0.011927 & $6.37 \mathrm{E}-25$ & 0.847905 & 0.071561 & 0.020881 & 0.030212 & $5.33 \mathrm{E}-08$ & 0.014247 & 0.003263 \\
2 & 0.013917 & $6.16 \mathrm{E}-25$ & 0.856949 & 0.065952 & 0.019238 & 0.027813 & $5.37 \mathrm{E}-08$ & 0.013122 & 0.003005 \\
3 & 0.016586 & $5.88 \mathrm{E}-25$ & 0.854767 & 0.065701 & 0.019165 & 0.02771 & $5.43 \mathrm{E}-08$ & 0.013073 & 0.002994 \\
4 & 0.020114 & $5.54 \mathrm{E}-25$ & 0.851722 & 0.065454 & 0.019094 & 0.027606 & $5.50 \mathrm{E}-08$ & 0.013024 & 0.002983 \\
5 & 0.024707 & $5.15 \mathrm{E}-25$ & 0.847756 & 0.065134 & 0.019 & 0.027471 & $5.60 \mathrm{E}-08$ & 0.01296 & 0.002968 \\
6 & 0.030573 & $4.73 \mathrm{E}-25$ & 0.842691 & 0.064724 & 0.018881 & 0.027299 & $5.72 \mathrm{E}-08$ & 0.012879 & 0.002949 \\
7 & 0.03789 & $4.28 \mathrm{E}-25$ & 0.836371 & 0.064214 & 0.018732 & 0.027084 & $5.88 \mathrm{E}-08$ & 0.012777 & 0.002926 \\
8 & 0.046763 & $3.83 \mathrm{E}-25$ & 0.828707 & 0.063596 & 0.018552 & 0.026823 & $6.07 \mathrm{E}-08$ & 0.012654 & 0.002898 \\
9 & 0.057172 & $3.42 \mathrm{E}-25$ & 0.819714 & 0.062872 & 0.01834 & 0.026518 & $6.30 \mathrm{E}-08$ & 0.01251 & 0.002865 \\
10 & 0.06895 & $3.03 \mathrm{E}-25$ & 0.809533 & 0.062052 & 0.018101 & 0.026173 & $6.49 \mathrm{E}-08$ & 0.012347 & 0.002828 \\
\hline
\end{tabular}


Table S9(d) Stream results of the flowsheet for IL-based Decarbonization (two single-stage)

\begin{tabular}{|c|c|c|c|c|c|c|c|c|c|c|c|}
\hline & 1 & 2 & 3 & 4 & 5 & 8 & ACIDGAS & $\begin{array}{c}\mathrm{Bmim} \\
\mathrm{Tf}_{2} \mathrm{~N}\end{array}$ & GASIN & GASOUT & RICHOUT \\
\hline Temperature ${ }^{\circ} \mathrm{C}$ & 22.37 & 24.82 & 24.82 & 20.00 & 27.00 & 20.00 & 27.00 & 20.00 & 20.00 & 23.16 & 25.47 \\
\hline Pressure bar & 60.00 & 15.00 & 15.00 & 60.00 & 1.00 & 60.00 & 1.00 & 1.00 & 60.00 & 60.00 & 60.00 \\
\hline Vapor Frac & 0.00 & 1.00 & 0.00 & 1.00 & 0.00 & 1.00 & 1.00 & 0.00 & 1.00 & 1.00 & 0.00 \\
\hline Mole Flow kmol/hr & 1460.50 & 155.83 & 1622.13 & 2155.83 & 1460.50 & 155.83 & 161.64 & 1460.50 & 2000.00 & 1838.36 & 1777.97 \\
\hline Mass Flow kg/hr & 601328.23 & 3543.32 & 607235.31 & 44581.88 & 601328.23 & 3543.32 & 5907.09 & 601328.23 & 41038.56 & 35131.47 & 610778.63 \\
\hline Volume Flow cum $/ \mathrm{hr}$ & 307.97 & 257.37 & 331.09 & 875.75 & 309.03 & 63.30 & 4033.74 & 307.43 & 812.45 & 754.83 & 353.00 \\
\hline Enthalpy Gcal/hr & -56.25 & -5.60 & -68.18 & -51.96 & -55.30 & -5.61 & -11.86 & -56.76 & -46.35 & -34.43 & -73.78 \\
\hline \multicolumn{12}{|l|}{ Mass Flow kg/hr } \\
\hline $\mathrm{CO}_{2}$ & 1191.88 & 1632.81 & 6388.00 & 7793.88 & 1191.88 & 1632.81 & 5196.12 & 1191.88 & 6161.07 & 964.94 & 8020.81 \\
\hline $\mathrm{BmimTf}_{2} \mathrm{~N}$ & 600079.59 & 0.00 & 600079.59 & 0.00 & 600079.59 & 0.00 & 0.00 & 600079.59 & 0.00 & 0.00 & 600079.59 \\
\hline $\mathrm{CH}_{4}$ & 15.51 & 1899.96 & 703.83 & 27595.05 & 15.51 & 1899.96 & 688.33 & 15.51 & 25695.09 & 25006.76 & 2603.79 \\
\hline $\mathrm{C}_{2} \mathrm{H}_{6}$ & 0.00 & 5.69 & 0.03 & 3961.53 & 0.00 & 5.69 & 0.03 & 0.00 & 3955.84 & 3955.81 & 5.72 \\
\hline $\mathrm{C}_{3} \mathrm{H}_{8}$ & 0.00 & 1.97 & 0.01 & 1694.71 & 0.00 & 1.97 & 0.01 & 0.00 & 1692.74 & 1692.73 & 1.98 \\
\hline $\mathrm{N}_{2}$ & 0.00 & 0.62 & 0.00 & 1556.53 & 0.62 & 0.00 & 0.00 & 1555.91 & 1555.91 & 0.63 & 0.00 \\
\hline $\mathrm{H}_{2} \mathrm{O}$ & 3.22 & 0.00 & 3.24 & 0.02 & 0.00 & 0.01 & 3.22 & 0.02 & 0.00 & 3.24 & 0.00 \\
\hline $\mathrm{C}_{4} \mathrm{H}_{10}$ & 0.00 & 0.73 & 0.00 & 1523.06 & 0.73 & 0.00 & 0.00 & 1522.32 & 1522.32 & 0.74 & 0.00 \\
\hline $\mathrm{C}_{5} \mathrm{H}_{12}$ & 0.00 & 0.21 & 0.00 & 432.97 & 0.21 & 0.00 & 0.00 & 432.76 & 432.76 & 0.21 & 0.00 \\
\hline
\end{tabular}




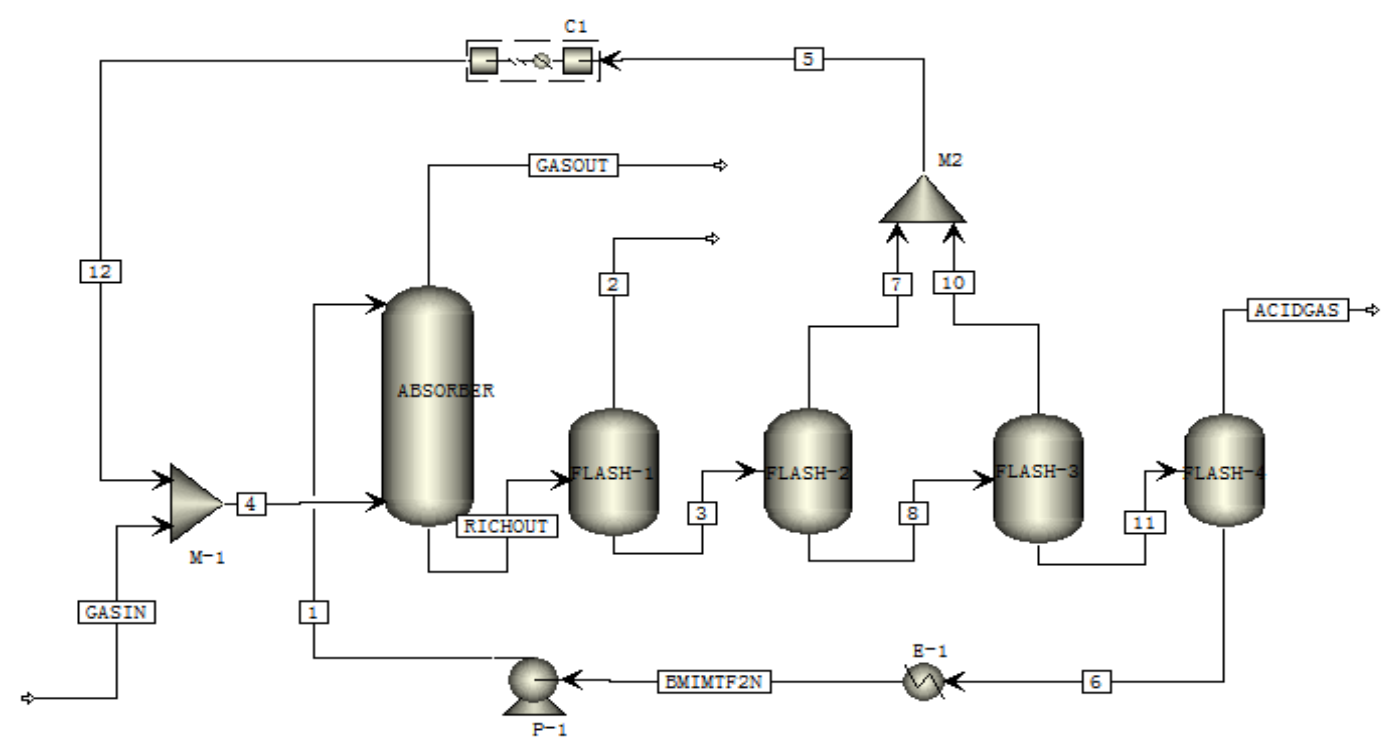

Figure S3 IL-based decarbonization flow sheet (Multi-stage flash)

Table S10(a) Operation parameters of key equipment in IL-based decarbonization process (Multi-stage flash)

\begin{tabular}{lll}
\hline Items & Unit & Value \\
\hline Absorber & & \\
Pressure of the column bottom & bar & 60 \\
Gas inlet temperature & ${ }^{\circ} \mathrm{C}$ & 20 \\
Gas outlet temperature & ${ }^{\circ} \mathrm{C}$ & 23.6 \\
Stage number & & 10 \\
Flash-1 & & \\
Pressure & bar & 55 \\
Temperature & ${ }^{\circ} \mathrm{C}$ & 25 \\
Flash-2 & & \\
Pressure & bar & 40 \\
Temperature & ${ }^{\circ} \mathrm{C}$ & 25 \\
Flash-3 & & \\
Pressure & bar & 30 \\
Temperature & ${ }^{\circ} \mathrm{C}$ & 25 \\
Flash-4 & & \\
Pressure & bar & 0.4 \\
Temperature & ${ }^{\circ} \mathrm{C}$ & 25 \\
Heat exchanger $(\mathrm{E}-1)$ & & 25 \\
Inlet temperature & ${ }^{\circ} \mathrm{C}$ & 20 \\
Outlet temperature & ${ }^{\circ} \mathrm{C}$ & 0.844 \\
Purified shale gas & & \\
$\mathrm{CH}_{4}$ & mole fraction & \\
CO & mole fraction & \\
\hline
\end{tabular}


Table S10(b) Temperature, Pressure and Vapor and Liquid Flow Rate Profiles of the Absorber of the IL-based decarbonization Process (Multi-stage flash)

\begin{tabular}{ccccc}
\hline Stage & $\begin{array}{c}\text { Temperature } \\
\mathrm{C}\end{array}$ & $\begin{array}{c}\text { Pressure } \\
\text { bar }\end{array}$ & $\begin{array}{c}\text { Liquid from (Mole) } \\
\mathrm{kmol} / \mathrm{hr}\end{array}$ & $\begin{array}{c}\text { Vapor from (Mole) } \\
\mathrm{kmol} / \mathrm{hr}\end{array}$ \\
\hline 1 & 23.58 & 59.98 & 24687.79 & 40532.01 \\
2 & 24.02 & 59.99 & 25125.04 & 43661.93 \\
3 & 24.42 & 60.01 & 25511.69 & 44099.18 \\
4 & 24.78 & 60.02 & 25862.13 & 44485.83 \\
5 & 25.10 & 60.03 & 26171.34 & 44836.27 \\
6 & 25.36 & 60.04 & 26437.9 & 45145.48 \\
7 & 25.59 & 60.05 & 26663.25 & 45412.04 \\
8 & 25.78 & 60.06 & 26850.45 & 45637.38 \\
9 & 25.97 & 60.07 & 27002.22 & 45824.59 \\
10 & 26.28 & 60.08 & 27143.92 & 45976.35 \\
\hline
\end{tabular}

Table S10(c) Vapor and Liquid Composition Profiles of the Absorber of the IL-based Decarbonization Process (Multi-stage flash)

Vapor (mole fraction)

\begin{tabular}{rccccccccc}
\hline Stage & $\mathrm{CO}_{2}$ & BMIMTF$_{2} \mathrm{~N}$ & $\mathrm{CH}_{4}$ & $\mathrm{C}_{2} \mathrm{H}_{6}$ & $\mathrm{C}_{3} \mathrm{H}_{8}$ & $\mathrm{~N}_{2}$ & $\mathrm{H}_{2} \mathrm{O}$ & $\mathrm{C}_{4} \mathrm{H}_{10}$ & $\mathrm{C}_{5} \mathrm{H}_{12}$ \\
\hline 1 & 0.0134 & $5.46 \mathrm{E}-25$ & 0.8442 & 0.0727 & 0.0212 & 0.0307 & $2.42 \mathrm{E}-08$ & 0.0145 & 0.0033 \\
2 & 0.0225 & $4.76 \mathrm{E}-25$ & 0.8451 & 0.0676 & 0.0197 & 0.0285 & $2.50 \mathrm{E}-08$ & 0.0135 & 0.0031 \\
3 & 0.0312 & $4.22 \mathrm{E}-25$ & 0.8378 & 0.0669 & 0.0195 & 0.0282 & $2.59 \mathrm{E}-08$ & 0.0133 & 0.0030 \\
4 & 0.0392 & $3.82 \mathrm{E}-25$ & 0.8309 & 0.0663 & 0.0193 & 0.0280 & $2.67 \mathrm{E}-08$ & 0.0132 & 0.0030 \\
5 & 0.0464 & $3.52 \mathrm{E}-25$ & 0.8248 & 0.0658 & 0.0192 & 0.0278 & $2.74 \mathrm{E}-08$ & 0.0131 & 0.0030 \\
6 & 0.0526 & $3.30 \mathrm{E}-25$ & 0.8194 & 0.0654 & 0.0191 & 0.0276 & $2.81 \mathrm{E}-08$ & 0.0130 & 0.0030 \\
7 & 0.0579 & $3.13 \mathrm{E}-25$ & 0.8149 & 0.0650 & 0.0190 & 0.0274 & $2.86 \mathrm{E}-08$ & 0.0129 & 0.0030 \\
8 & 0.0624 & $3.00 \mathrm{E}-25$ & 0.8110 & 0.0646 & 0.0189 & 0.0273 & $2.91 \mathrm{E}-08$ & 0.0129 & 0.0029 \\
9 & 0.0660 & $2.90 \mathrm{E}-25$ & 0.8079 & 0.0644 & 0.0188 & 0.0272 & $2.96 \mathrm{E}-08$ & 0.0128 & 0.0029 \\
10 & 0.0690 & $2.82 \mathrm{E}-25$ & 0.8054 & 0.0642 & 0.0187 & 0.0271 & $3.03 \mathrm{E}-08$ & 0.0128 & 0.0029 \\
\hline
\end{tabular}


Liquid (mole fraction)

\begin{tabular}{rcrccccccc}
\hline Stage & $\mathrm{CO}_{2}$ & BMIMTF$_{2} \mathrm{~N}$ & $\mathrm{CH}_{4}$ & $\mathrm{C}_{2} \mathrm{H}_{6}$ & $\mathrm{C}_{3} \mathrm{H}_{8}$ & $\mathrm{~N}_{2}$ & $\mathrm{H}_{2} \mathrm{O}$ & $\mathrm{C}_{4} \mathrm{H}_{10}$ & $\mathrm{C}_{5} \mathrm{H}_{12}$ \\
\hline 1 & 0.0239 & 0.8662 & 0.1091 & 0.000138 & $3.27 \mathrm{E}-05$ & $2.01 \mathrm{E}-05$ & 0.000169 & $1.75 \mathrm{E}-05$ & $4.00 \mathrm{E}-06$ \\
2 & 0.0392 & 0.8512 & 0.1090 & 0.000125 & $2.97 \mathrm{E}-05$ & $1.83 \mathrm{E}-05$ & 0.000166 & $1.58 \mathrm{E}-05$ & $3.62 \mathrm{E}-06$ \\
3 & 0.0530 & 0.8383 & 0.1081 & 0.000121 & $2.87 \mathrm{E}-05$ & $1.78 \mathrm{E}-05$ & 0.000163 & $1.53 \mathrm{E}-05$ & $3.50 \mathrm{E}-06$ \\
4 & 0.0652 & 0.8269 & 0.1072 & 0.000118 & $2.79 \mathrm{E}-05$ & $1.74 \mathrm{E}-05$ & 0.000161 & $1.49 \mathrm{E}-05$ & $3.40 \mathrm{E}-06$ \\
5 & 0.0757 & 0.8171 & 0.1065 & 0.000115 & $2.72 \mathrm{E}-05$ & $1.71 \mathrm{E}-05$ & 0.000159 & $1.45 \mathrm{E}-05$ & $3.32 \mathrm{E}-06$ \\
6 & 0.0846 & 0.8089 & 0.1059 & 0.000113 & $2.67 \mathrm{E}-05$ & $1.68 \mathrm{E}-05$ & 0.000157 & $1.42 \mathrm{E}-05$ & $3.25 \mathrm{E}-06$ \\
7 & 0.0920 & 0.8021 & 0.1053 & 0.000111 & $2.62 \mathrm{E}-05$ & $1.66 \mathrm{E}-05$ & 0.000156 & $1.39 \mathrm{E}-05$ & $3.19 \mathrm{E}-06$ \\
8 & 0.0980 & 0.7965 & 0.1049 & 0.00011 & $2.58 \mathrm{E}-05$ & $1.64 \mathrm{E}-05$ & 0.000155 & $1.37 \mathrm{E}-05$ & $3.14 \mathrm{E}-06$ \\
9 & 0.1029 & 0.7920 & 0.1045 & 0.000108 & $2.55 \mathrm{E}-05$ & $1.62 \mathrm{E}-05$ & 0.000154 & $1.35 \mathrm{E}-05$ & $3.10 \mathrm{E}-06$ \\
10 & 0.1067 & 0.7879 & 0.1043 & 0.000107 & $2.52 \mathrm{E}-05$ & $1.61 \mathrm{E}-05$ & 0.000154 & $1.34 \mathrm{E}-05$ & $3.06 \mathrm{E}-06$ \\
\hline
\end{tabular}


Table S10(d) Stream results of the flowsheet for IL-based Decarbonization (Multi-stage flash)

\begin{tabular}{|c|c|c|c|c|c|c|c|c|c|c|c|c|c|c|c|c|c|}
\hline & 1 & 2 & 3 & 4 & 5 & 6 & 7 & 8 & 9 & 10 & 11 & 12 & ACIDGAS & BMIMTf $_{2} \mathrm{~N}$ & GASIN & GASOUT & RICHOUT \\
\hline Temperature $\mathrm{C}$ & 22.20 & 25.00 & 25.00 & 20.00 & 25.00 & 25.00 & 25.00 & 25.00 & 25.03 & 25.00 & 25.00 & 20.00 & 25.00 & 20.00 & 20.00 & 23.55 & 26.27 \\
\hline Pressure bar & 60.00 & 55.00 & 55.00 & 60.00 & 30.00 & 0.40 & 40.00 & 40.00 & 1.00 & 30.00 & 30.00 & 60.00 & 0.40 & 1.00 & 60.00 & 60.00 & 60.00 \\
\hline Vapor Frac & 0.00 & 1.00 & 0.00 & 1.00 & 1.00 & 0.00 & 1.00 & 0.00 & 0.00 & 1.00 & 0.00 & 1.00 & 1.00 & 0.00 & 1.00 & 1.00 & 0.00 \\
\hline Mole Flow scmh & 21557.88 & 36.09 & 27108.87 & 46118.31 & 1291.08 & 21557.88 & 671.39 & 26437.49 & 21557.88 & 619.70 & 25817.79 & 1291.08 & 4259.92 & 21557.88 & 44827.22 & 40531.20 & 27144.96 \\
\hline Mass Flow kg/hr & 400445.41 & 31.08 & 407856.97 & 42153.84 & 1115.27 & 400445.41 & 567.37 & 407289.61 & 400445.41 & 547.90 & 406741.75 & 1115.27 & 6296.52 & 400445.41 & 41038.56 & 34710.94 & 407888.05 \\
\hline Volume Flow cum/sec & 0.06 & 0.00 & 0.07 & 0.23 & 0.01 & 0.06 & 0.01 & 0.07 & 0.06 & 0.01 & 0.06 & 0.01 & 3.27 & 0.06 & 0.23 & 0.21 & 0.07 \\
\hline Enthalpy Gcal/hr & -36.41 & -0.04 & -50.43 & -47.89 & -1.54 & -36.01 & -0.77 & -49.66 & -36.01 & -0.78 & -48.87 & -1.54 & -12.19 & -36.75 & -46.35 & -34.06 & -50.25 \\
\hline \multicolumn{18}{|l|}{ Mass Flow kg/hr } \\
\hline $\mathrm{CO}_{2}$ & 294.27 & 5.21 & 5683.44 & 6458.97 & 297.90 & 294.27 & 133.81 & 5549.64 & 294.27 & 164.10 & 5385.54 & 297.90 & 5091.26 & 294.27 & 6161.07 & 1064.58 & 5688.65 \\
\hline BMIMTF $_{2} \mathrm{~N}$ & 400128.42 & 0.00 & 400128.17 & 0.00 & 0.00 & 400128.42 & 0.00 & 400128.18 & 400128.42 & 0.00 & 400128.22 & 0.00 & 0.00 & 400128.42 & 0.00 & 0.00 & 400128.17 \\
\hline $\mathrm{CH}_{4}$ & 7.26 & 22.33 & 2003.59 & 26509.00 & 813.91 & 7.26 & 430.24 & 1573.35 & 7.26 & 383.67 & 1189.68 & 813.91 & 1182.42 & 7.26 & 25695.09 & 24490.34 & 2025.92 \\
\hline $\mathrm{C}_{2} \mathrm{H}_{6}$ & 0.00 & 1.78 & 2.12 & 3957.96 & 2.12 & 0.00 & 2.03 & 0.09 & 0.00 & 0.09 & 0.00 & 2.12 & 0.00 & 0.00 & 3955.84 & 3954.05 & 3.91 \\
\hline $\mathrm{C}_{3} \mathrm{H}_{8}$ & 0.00 & 0.69 & 0.66 & 1693.40 & 0.66 & 0.00 & 0.64 & 0.02 & 0.00 & 0.02 & 0.00 & 0.66 & 0.00 & 0.00 & 1692.74 & 1692.05 & 1.35 \\
\hline $\mathrm{N}_{2}$ & 0.00 & 0.39 & 0.16 & 1556.07 & 0.16 & 0.00 & 0.16 & 0.00 & 0.00 & 0.00 & 0.00 & 0.16 & 0.00 & 0.00 & 1555.91 & 1555.52 & 0.55 \\
\hline $\mathrm{H}_{2} \mathrm{O}$ & 3.34 & 0.00 & 3.36 & 0.02 & 0.00 & 3.34 & 0.00 & 3.36 & 3.34 & 0.00 & 3.36 & 0.00 & 0.02 & 3.34 & 0.02 & 0.00 & 3.36 \\
\hline $\mathrm{C}_{4} \mathrm{H}_{10}$ & 0.00 & 0.54 & 0.40 & 1522.73 & 0.40 & 0.00 & 0.39 & 0.01 & 0.00 & 0.01 & 0.00 & 0.40 & 0.00 & 0.00 & 1522.32 & 1521.78 & 0.94 \\
\hline $\mathrm{C}_{5} \mathrm{H}_{12}$ & 0.00 & 0.15 & 0.11 & 432.87 & 0.11 & 0.00 & 0.11 & 0.00 & 0.00 & 0.00 & 0.00 & 0.11 & 0.00 & 0.00 & 432.76 & 432.60 & 0.27 \\
\hline
\end{tabular}




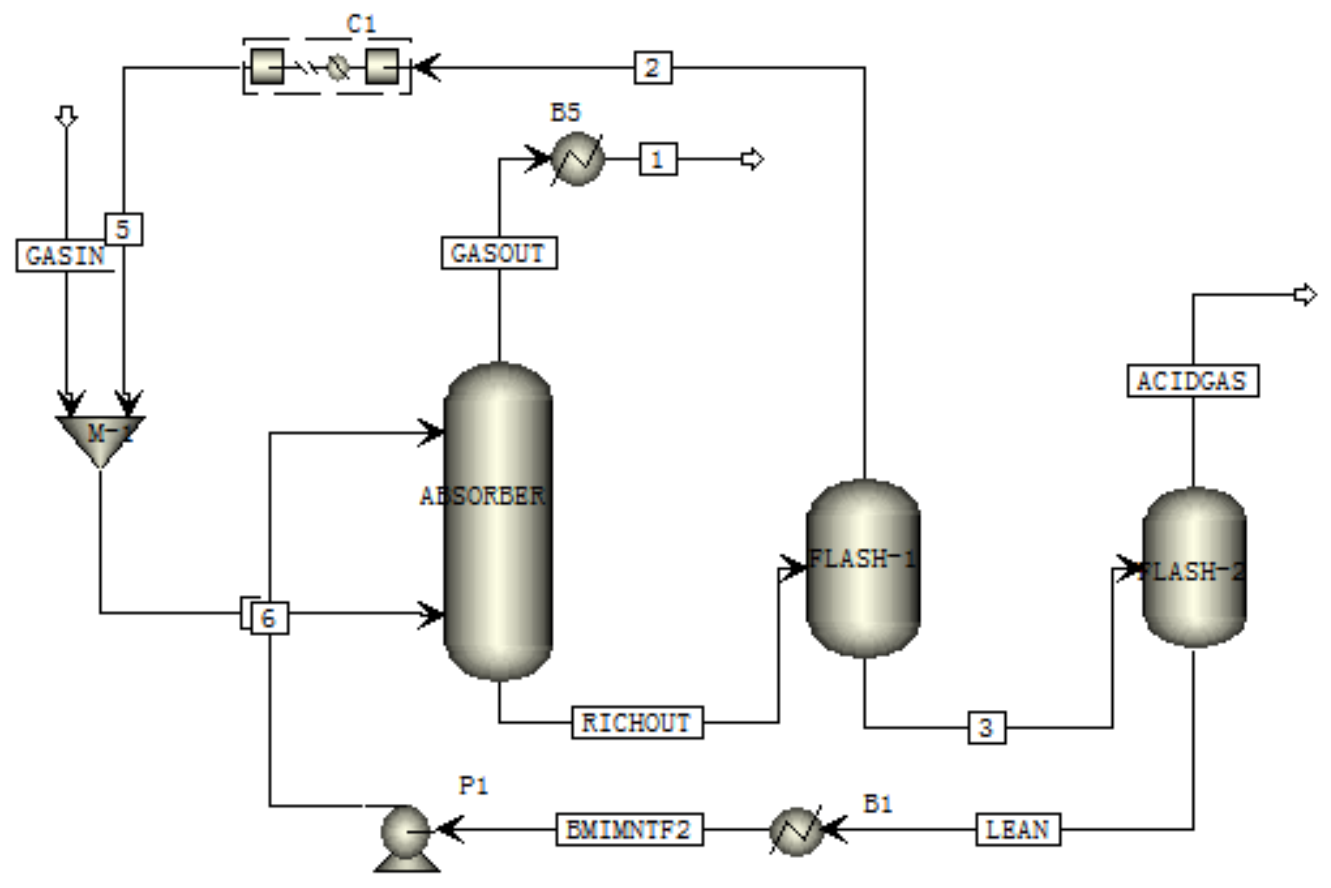

Figure S4 Flowsheet of different raw gas component for IL-based Decarbonization (two single-stage) 
Table S11 Stream results of the flowsheet for IL-based Decarbonization (two single-stage) (raw gas $\mathrm{V}_{\mathrm{CO} 2}: \mathrm{V}_{\mathrm{CH} 4}=20 \%: 80 \%$ )

\begin{tabular}{|c|c|c|c|c|c|c|c|c|c|c|c|c|}
\hline & 1 & 2 & 3 & 4 & 5 & 6 & ACIDGAS & BMIMNTF2 & GASIN & GASOUT & LEAN & RICHOUT \\
\hline Temperature $\mathrm{C}$ & 0 & 25.9 & 25.9 & 21.1 & 30 & 21.8 & 27 & 20 & 20 & 22.5 & 27 & 27.1 \\
\hline Pressure bar & 1 & 15 & 15 & 40 & 40 & 40 & 1 & 1 & 40 & 40 & 1 & 40 \\
\hline Vapor Frac & 1 & 1 & 0 & 1 & 1 & 0 & 1 & 0 & 1 & 1 & 0 & 0 \\
\hline Mole Flow kmol/hr & 1600.159 & 242.817 & 2473.163 & 2242.817 & 242.817 & 2073.312 & 399.851 & 2073.312 & 2000 & 1600.159 & 2073.312 & 2715.981 \\
\hline Mass Flow kg/hr & 27028.16 & 7115.944 & 868269.8 & 50388.28 & 7115.944 & 852025.2 & 16244.63 & 852025.2 & 43272.34 & 27028.16 & 852025.2 & 875385.8 \\
\hline Volume Flow cum/hr & 36340.57 & 402.49 & 489.586 & 1371.757 & 153.005 & 436.694 & 9978.467 & 436.117 & 1218.673 & 983.355 & 438.388 & 519.746 \\
\hline Enthalpy $\quad \mathrm{Gcal} / \mathrm{hr}$ & -32.515 & -13.093 & -114.338 & -79.244 & -13.085 & -80.394 & -33.889 & -80.893 & -66.159 & -32.212 & -78.952 & -127.432 \\
\hline \multicolumn{13}{|l|}{ Mass Flow kg/hr } \\
\hline $\mathrm{CO}_{2}$ & 2135.712 & 5067.851 & 17483.81 & 22671.77 & 5067.851 & 2015.157 & 15468.66 & 2015.157 & 17603.92 & 2135.712 & 2015.157 & 22551.66 \\
\hline $\mathrm{BMIMTF}_{2} \mathrm{~N}$ & trace & trace & 850000 & trace & trace & 850000 & trace & 850000 & & trace & 850000 & 850000 \\
\hline $\mathrm{CH}_{4}$ & 24892.45 & 2048.093 & 786.005 & 27716.51 & 2048.093 & 10.034 & 775.971 & 10.034 & 25668.42 & 24892.45 & 10.034 & 2834.097 \\
\hline \multicolumn{13}{|l|}{ Mass Frac } \\
\hline $\mathrm{CO}_{2}$ & 0.079 & 0.712 & 0.02 & 0.45 & 0.712 & 0.002 & 0.952 & 0.002 & 0.407 & 0.079 & 0.002 & 0.026 \\
\hline $\mathrm{BMIMTF}_{2} \mathrm{~N}$ & trace & trace & 0.979 & trace & trace & 0.998 & trace & 0.998 & & trace & 0.998 & 0.971 \\
\hline $\mathrm{CH}_{4}$ & 0.921 & 0.288 & 905 PPM & 0.55 & 0.288 & 12 PPM & 0.048 & $12 \mathrm{PPM}$ & 0.593 & 0.921 & 12 PPM & 0.003 \\
\hline \multicolumn{13}{|l|}{ Mole Flow kmol/hr } \\
\hline $\mathrm{CO}_{2}$ & 48.528 & 115.153 & 397.271 & 515.153 & 115.153 & 45.789 & 351.482 & 45.789 & 400 & 48.528 & 45.789 & 512.424 \\
\hline $\mathrm{BMIMTF}_{2} \mathrm{~N}$ & trace & trace & 2026.898 & trace & trace & 2026.898 & trace & 2026.898 & & trace & 2026.898 & 2026.898 \\
\hline $\mathrm{CH}_{4}$ & 1551.631 & 127.665 & 48.994 & 1727.665 & 127.665 & 0.625 & 48.369 & 0.625 & 1600 & 1551.631 & 0.625 & 176.659 \\
\hline \multicolumn{13}{|l|}{ Mole Frac } \\
\hline $\mathrm{CO}_{2}$ & 0.03 & 0.474 & 0.161 & 0.23 & 0.474 & 0.022 & 0.879 & 0.022 & 0.2 & 0.03 & 0.022 & 0.189 \\
\hline $\mathrm{BMIMTF}_{2} \mathrm{~N}$ & trace & trace & 0.82 & trace & trace & 0.978 & trace & 0.978 & & trace & 0.978 & 0.746 \\
\hline $\mathrm{CH}_{4}$ & 0.97 & 0.526 & 0.02 & 0.77 & 0.526 & 302 PPM & 0.121 & 302 PPM & 0.8 & 0.97 & 302 PPM & 0.065 \\
\hline
\end{tabular}


Table S12 Stream results of the flowsheet for IL Decarbonization (two single-stage) (raw gas $\mathrm{V}_{\mathrm{CO} 2}: \mathrm{V}_{\mathrm{CH} 4}=40 \%: 60 \%$ )

\begin{tabular}{|c|c|c|c|c|c|c|c|c|c|c|c|c|}
\hline & 1 & 2 & 3 & 4 & 5 & 6 & ACIDGAS & BMIMNTF2 & GASIN & GASOUT & LEANOUT & RICHOUT \\
\hline Temperature $\mathrm{C}$ & 0 & 28.4 & 28.4 & 23 & 30 & 21.8 & 27 & 20 & 20 & 22.4 & 27 & 32.7 \\
\hline Pressure bar & 1 & 15 & 15 & 40 & 40 & 40 & 1 & 1 & 40 & 40 & 1 & 40 \\
\hline Vapor Frac & 1 & 1 & 0 & 1 & 1 & 0 & 1 & 0 & 1 & 1 & 0 & 0 \\
\hline Mole Flow kmol/hr & 1207.131 & 819.992 & 3481.167 & 2819.992 & 819.992 & 2688.301 & 792.866 & 2688.301 & 2000 & 1207.131 & 2688.301 & 4301.159 \\
\hline Mass Flow kg/hr & 20393.48 & 31349.72 & 1136980 & 85808.87 & 31349.72 & 1102910 & 34065.79 & 1102910 & 54459.15 & 20393.48 & 1102910 & 1168330 \\
\hline Volume Flow cum $/ \mathrm{hr}$ & 27414.67 & 1370.494 & 663.962 & 1735.646 & 516.695 & 565.831 & 19786.34 & 565.099 & 974.938 & 741.612 & 568.039 & 746.371 \\
\hline Enthalpy $\quad \mathrm{Gcal} / \mathrm{hr}$ & -24.54 & -64.138 & -176.904 & -160.762 & -64.126 & -104.594 & -72.25 & -105.229 & -96.636 & -24.312 & -102.714 & -241.042 \\
\hline \multicolumn{13}{|l|}{ Mass Flow kg/hr } \\
\hline $\mathrm{CO}_{2}$ & 1617.317 & 28631.89 & 36446.06 & 63839.73 & 28631.89 & 2855.123 & 33590.78 & 2855.123 & 35207.84 & 1617.317 & 2855.123 & 65077.94 \\
\hline $\mathrm{BMIMTF}_{2} \mathrm{~N}$ & trace & trace & 1100050 & trace & trace & 1100060 & trace & 1100060 & & trace & 1100060 & 1100050 \\
\hline $\mathrm{CH}_{4}$ & 18776.16 & 2717.835 & 479.042 & 21969.15 & 2717.835 & 4.025 & 475.014 & 4.025 & 19251.31 & 18776.16 & 4.025 & 3196.877 \\
\hline \multicolumn{13}{|l|}{ Mass Frac } \\
\hline $\mathrm{CO}_{2}$ & 0.079 & 0.913 & 0.032 & 0.744 & 0.913 & 0.003 & 0.986 & 0.003 & 0.646 & 0.079 & 0.003 & 0.056 \\
\hline $\mathrm{BMIMTF}_{2} \mathrm{~N}$ & trace & trace & 0.968 & trace & trace & 0.997 & trace & 0.997 & & trace & 0.997 & 0.942 \\
\hline $\mathrm{CH}_{4}$ & 0.921 & 0.087 & 421 PPM & 0.256 & 0.087 & 4 PPM & 0.014 & 4 PPM & 0.354 & 0.921 & 4 PPM & 0.003 \\
\hline \multicolumn{13}{|l|}{ Mole Flow kmol/hr } \\
\hline $\mathrm{CO}_{2}$ & 36.749 & 650.58 & 828.135 & 1450.58 & 650.58 & 64.875 & 763.257 & 64.875 & 800 & 36.749 & 64.875 & 1478.715 \\
\hline $\mathrm{BMIMTF}_{2} \mathrm{~N}$ & trace & trace & 2623.172 & trace & trace & 2623.176 & trace & 2623.176 & & trace & 2623.176 & 2623.172 \\
\hline $\mathrm{CH}_{4}$ & 1170.382 & 169.412 & 29.86 & 1369.412 & 169.412 & 0.251 & 29.609 & 0.251 & 1200 & 1170.382 & 0.251 & 199.272 \\
\hline \multicolumn{13}{|l|}{ Mole Frac } \\
\hline $\mathrm{CO}_{2}$ & 0.03 & 0.793 & 0.238 & 0.514 & 0.793 & 0.024 & 0.963 & 0.024 & 0.4 & 0.03 & 0.024 & 0.344 \\
\hline $\mathrm{BMIMTF}_{2} \mathrm{~N}$ & trace & trace & 0.754 & trace & trace & 0.976 & trace & 0.976 & & trace & 0.976 & 0.61 \\
\hline $\mathrm{CH}_{4}$ & 0.97 & 0.207 & 0.009 & 0.486 & 0.207 & 93 PPM & 0.037 & 93 PPM & 0.6 & 0.97 & 93 PPM & 0.046 \\
\hline
\end{tabular}


Table S13 Stream results of the flowsheet for IL-based Decarbonization (two single-stage) (raw gas $\mathrm{V}_{\mathrm{CO} 2}: \mathrm{V}_{\mathrm{CH} 4}=60 \%: 40 \%$ )

\begin{tabular}{|c|c|c|c|c|c|c|c|c|c|c|c|c|}
\hline & 1 & 2 & 3 & 4 & 5 & 6 & ACIDGAS & BMIMNTF2 & GASIN & GASOUT & LEANOUT & RICHOUT \\
\hline Temperature $\mathrm{C}$ & 0 & 29.2 & 29.2 & 24.8 & 30 & 21.7 & 27 & 20 & 20 & 22.3 & 27 & 36.5 \\
\hline Pressure bar & 1 & 15 & 15 & 40 & 40 & 40 & 1 & 1 & 40 & 40 & 1 & 40 \\
\hline Vapor Frac & 1 & 1 & 0 & 1 & 1 & 0 & 1 & 0 & 1 & 1 & 0 & 0 \\
\hline Mole Flow kmol/hr & 804.823 & 1835.195 & 4823.548 & 3835.195 & 1835.195 & 3628.381 & 1195.168 & 3628.381 & 2000 & 804.823 & 3628.381 & 6658.743 \\
\hline Mass Flow kg/hr & 13573.44 & 76099.45 & 1540030 & 141745.4 & 76099.45 & 1487960 & 52071.61 & 1487960 & 65645.97 & 13573.44 & 1487960 & 1616130 \\
\hline Volume Flow cum $/ \mathrm{hr}$ & 18278 & 3075.402 & 908.545 & 2375.46 & 1156.397 & 763.548 & 29825.97 & 762.577 & 974.938 & 494.319 & 766.544 & 1074.938 \\
\hline Enthalpy $\quad \mathrm{Gcal} / \mathrm{hr}$ & -16.298 & -159.704 & -252.256 & -286.803 & -159.691 & -141.303 & -110.873 & -142.145 & -127.112 & -16.146 & -138.751 & -411.958 \\
\hline \multicolumn{13}{|l|}{ Mass Flow kg/hr } \\
\hline $\mathrm{CO}_{2}$ & 1041.532 & 73422.25 & 55706.66 & 126234 & 73422.25 & 3937.594 & 51769.03 & 3937.594 & 52811.76 & 1041.532 & 3937.594 & 129128.9 \\
\hline $\mathrm{BMIMTF}_{2} \mathrm{~N}$ & trace & trace & 1484020 & trace & trace & 1484020 & trace & 1484020 & & trace & 1484020 & 1484020 \\
\hline $\mathrm{CH}_{4}$ & 12531.91 & 2677.194 & 304.881 & 15511.4 & 2677.194 & 2.297 & 302.584 & 2.297 & 12834.21 & 12531.91 & 2.297 & 2982.075 \\
\hline \multicolumn{13}{|l|}{ Mass Frac } \\
\hline $\mathrm{CO}_{2}$ & 0.077 & 0.965 & 0.036 & 0.891 & 0.965 & 0.003 & 0.994 & 0.003 & 0.804 & 0.077 & 0.003 & 0.08 \\
\hline $\mathrm{BMIMTF}_{2} \mathrm{~N}$ & trace & trace & 0.964 & trace & trace & 0.997 & trace & 0.997 & & trace & 0.997 & 0.918 \\
\hline $\mathrm{CH}_{4}$ & 0.923 & 0.035 & 198 PPM & 0.109 & 0.035 & $2 \mathrm{PPM}$ & 0.006 & 2 PPM & 0.196 & 0.923 & $2 \mathrm{PPM}$ & 0.002 \\
\hline \multicolumn{13}{|l|}{ Mole Flow kmol/hr } \\
\hline $\mathrm{CO}_{2}$ & 23.666 & 1668.316 & 1265.778 & 2868.316 & 1668.316 & 89.471 & 1176.307 & 89.471 & 1200 & 23.666 & 89.471 & 2934.094 \\
\hline $\mathrm{BMIMTF}_{2} \mathrm{~N}$ & trace & trace & 3538.766 & trace & trace & 3538.767 & trace & 3538.767 & & trace & 3538.767 & 3538.766 \\
\hline $\mathrm{CH}_{4}$ & 781.157 & 166.879 & 19.004 & 966.879 & 166.879 & 0.143 & 18.861 & 0.143 & 800 & 781.157 & 0.143 & 185.883 \\
\hline \multicolumn{13}{|l|}{ Mole Frac } \\
\hline $\mathrm{CO}_{2}$ & 0.029 & 0.909 & 0.262 & 0.748 & 0.909 & 0.025 & 0.984 & 0.025 & 0.6 & 0.029 & 0.025 & 0.441 \\
\hline $\mathrm{BMIMTF}_{2} \mathrm{~N}$ & trace & trace & 0.734 & trace & trace & 0.975 & trace & 0.975 & & trace & 0.975 & 0.531 \\
\hline $\mathrm{CH}_{4}$ & 0.971 & 0.091 & 0.004 & 0.252 & 0.091 & 39 PPM & 0.016 & 39 PPM & 0.4 & 0.971 & 39 PPM & 0.028 \\
\hline
\end{tabular}


Table S14 Stream results of the flowsheet for IL-based Decarbonization (two single-stage) (raw gas $\mathrm{V}_{\mathrm{CO} 2}: \mathrm{V}_{\mathrm{CH} 4}=80 \%: 20 \%$ )

\begin{tabular}{|c|c|c|c|c|c|c|c|c|c|c|c|c|}
\hline & 1 & 2 & 3 & 4 & 5 & 6 & ACIDGAS & BMIMNTF2 & GASIN & GASOUT & LEANOUT & RICHOUT \\
\hline Temperature $\mathrm{C}$ & 0 & 29.5 & 29.5 & 26 & 30 & 21.7 & 27 & 20 & 20 & 22.3 & 27 & 38.7 \\
\hline Pressure bar & 1 & 15 & 15 & 40 & 40 & 40 & 1 & 1 & 40 & 40 & 1 & 40 \\
\hline Vapor Frac & 1 & 1 & 0 & 1 & 1 & 0 & 1 & 0 & 1 & 1 & 0 & 0 \\
\hline Mole Flow kmol/hr & 401.164 & 2917.118 & 6240.316 & 4917.118 & 2917.118 & 4641.52 & 1598.795 & 4641.52 & 2000 & 401.164 & 4641.52 & 9157.434 \\
\hline Mass Flow kg/hr & 6765.217 & 125223.2 & 1973150 & 202056 & 125223.2 & 1903090 & 70065.86 & 1903090 & 76832.78 & 6765.217 & 1903090 & 2098380 \\
\hline Volume Flow cum $/ \mathrm{hr}$ & 9110.671 & 4894.181 & 1168.99 & 3057.148 & 1838.141 & 976.664 & 39898.68 & 975.437 & 974.938 & 246.369 & 980.511 & 1420.777 \\
\hline Enthalpy $\quad \mathrm{Gcal} / \mathrm{hr}$ & -8.122 & -265.452 & -330.361 & -423.029 & -265.44 & -180.836 & -149.431 & -181.9 & -157.589 & -8.047 & -177.558 & -595.814 \\
\hline \multicolumn{13}{|l|}{ Mass Flow kg/hr } \\
\hline $\mathrm{CO}_{2}$ & 518.418 & 123411.3 & 74978.25 & 193827 & 123411.3 & 5082.637 & 69895.6 & 5082.637 & 70415.68 & 518.418 & 5082.637 & 198389.6 \\
\hline $\mathrm{BMIMTF}_{2} \mathrm{~N}$ & trace & trace & 1898000 & trace & trace & 1898000 & trace & 1898000 & & trace & 1898000 & 1898000 \\
\hline $\mathrm{CH}_{4}$ & 6246.799 & 1811.862 & 171.494 & 8228.966 & 1811.862 & 1.236 & 170.258 & 1.236 & 6417.104 & 6246.799 & 1.236 & 1983.356 \\
\hline \multicolumn{13}{|l|}{ Mass Frac } \\
\hline $\mathrm{CO}_{2}$ & 0.077 & 0.986 & 0.038 & 0.959 & 0.986 & 0.003 & 0.998 & 0.003 & 0.916 & 0.077 & 0.003 & 0.095 \\
\hline $\mathrm{BMIMTF}_{2} \mathrm{~N}$ & trace & trace & 0.962 & trace & trace & 0.997 & trace & 0.997 & & trace & 0.997 & 0.905 \\
\hline $\mathrm{CH}_{4}$ & 0.923 & 0.014 & 87 PPM & 0.041 & 0.014 & 650 PPB & 0.002 & $650 \mathrm{PPB}$ & 0.084 & 0.923 & $650 \mathrm{PPB}$ & 945 PPM \\
\hline \multicolumn{13}{|l|}{ Mole Flow kmol/hr } \\
\hline $\mathrm{CO}_{2}$ & 11.78 & 2804.179 & 1703.672 & 4404.179 & 2804.179 & 115.489 & 1588.183 & 115.489 & 1600 & 11.78 & 115.489 & 4507.85 \\
\hline $\mathrm{BMIMTF}_{2} \mathrm{~N}$ & trace & trace & 4525.954 & trace & trace & 4525.954 & trace & 4525.954 & & trace & 4525.954 & 4525.954 \\
\hline $\mathrm{CH}_{4}$ & 389.384 & 112.94 & 10.69 & 512.94 & 112.94 & 0.077 & 10.613 & 0.077 & 400 & 389.384 & 0.077 & 123.629 \\
\hline \multicolumn{13}{|l|}{ Mole Frac } \\
\hline $\mathrm{CO}_{2}$ & 0.029 & 0.961 & 0.273 & 0.896 & 0.961 & 0.025 & 0.993 & 0.025 & 0.8 & 0.029 & 0.025 & 0.492 \\
\hline BMIMTF $_{2} \mathrm{~N}$ & trace & trace & 0.725 & trace & trace & 0.975 & trace & 0.975 & & trace & 0.975 & 0.494 \\
\hline $\mathrm{CH}_{4}$ & 0.971 & 0.039 & 0.002 & 0.104 & 0.039 & 17 PPM & 0.007 & 17 PPM & 0.2 & 0.971 & 17 PPM & 0.014 \\
\hline
\end{tabular}

